\title{
Traveling-wave photomixers fabricated on high energy nitrogen-ion-implanted GaAs
}

M. Mikulics, E. A. Michael, M. Marso, M. Lepsa, A. van der Hart, H. Lüth, A. Dewald, S. Stanček, M. Mozolik, and P. Kordoš

Citation: Appl. Phys. Lett. 89, 071103 (2006);

View online: https://doi.org/10.1063/1.2337523

View Table of Contents: http://aip.scitation.org/toc/apl/89/7

Published by the American Institute of Physics

\section{Articles you may be interested in}

Traveling-wave photomixer with recessed interdigitated contacts on low-temperature-grown GaAs

Applied Physics Letters 88, 041118 (2006); 10.1063/1.2168250

Terahertz photomixing with diode lasers in low-temperature-grown GaAs

Applied Physics Letters 67, 3844 (1995); 10.1063/1.115292

Terahertz photonic mixers as local oscillators for hot electron bolometer and superconductor-insulatorsuperconductor astronomical receivers

Journal of Applied Physics 100, 043116 (2006); 10.1063/1.2336486

ErAs:GaAs photomixer with two-decade tunability and $12 \mu \mathrm{W}$ peak output power

Applied Physics Letters 85, 3983 (2004); 10.1063/1.1813635

Photomixing up to $3.8 \mathrm{THz}$ in low-temperature-grown GaAs

Applied Physics Letters 66, 285 (1995); 10.1063/1.113519

Efficient terahertz emission from ballistic transport enhanced $n-i-p-n-i-p$ superlattice photomixers Applied Physics Letters 90, 212115 (2007); 10.1063/1.2743400

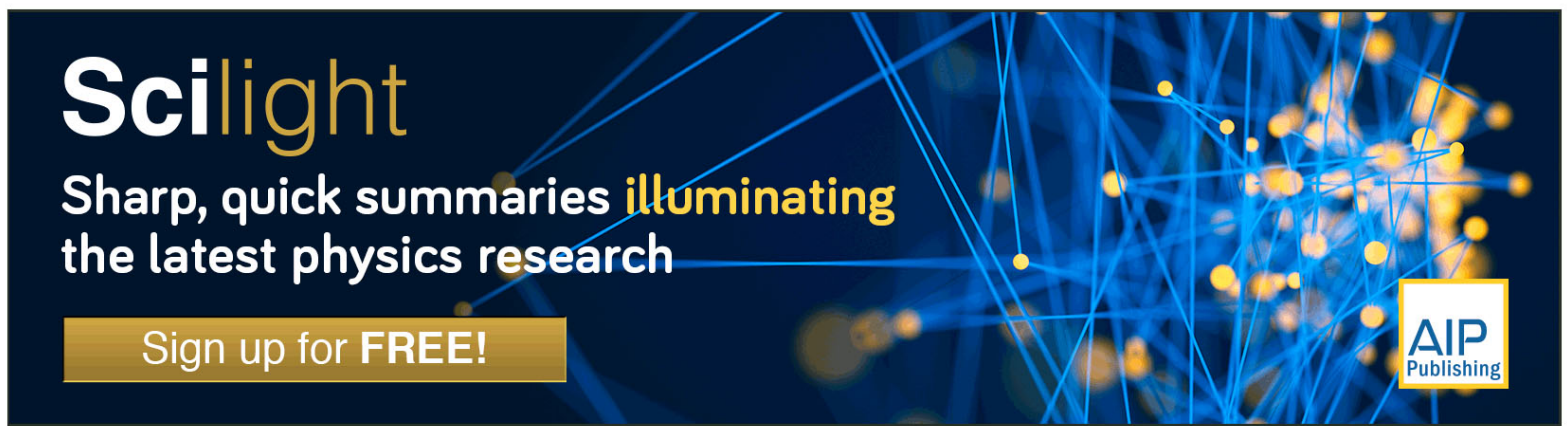




\title{
Traveling-wave photomixers fabricated on high energy nitrogen-ion-implanted GaAs
}

\author{
M. Mikulics ${ }^{\text {a) }}$ \\ Institut für Hochfrequenztechnik, Technische Universität Braunschweig, Schleinitzstraße 22, \\ D-38106 Braunschweig Germany and Institute of Bio- and Nanosystems, Research Center Jülich, \\ D-52425 Jülich, Germany \\ E. A. Michael \\ Physics Institute, University of Cologne, Zuelpicherstrasse 77, D-50937 Cologne, Germany \\ M. Marso, ${ }^{\text {b) }}$ M. Lepsa, A. van der Hart, and H. Lüth \\ Institute of Bio- and Nanosystems, Research Center Jülich, D-52425 Jülich, Germany
}

\begin{abstract}
A. Dewald
Institute of Nuclear Physics, University of Cologne, Zuelpicherstrasse 77, D-50937 Cologne, Germany

S. Stanček and M. Mozolik

Department of Nuclear Physics and Technology, Slovak University of Technology, SK-81219 Bratislava, Slovak Republic

P. Kordoš

Institute of Electrical Engineering, Slovak Academy of Sciences, SK-84104 Bratislava, Slovak Republic and Institute of Microelectronics, Slovak Technical University, SK-81219 Bratislava, Slovak Republic
\end{abstract}

(Received 2 May 2006; accepted 28 June 2006; published online 15 August 2006)

\begin{abstract}
The authors report on fabrication and measurement of traveling-wave photomixers based on high energy and low dose nitrogen-ion-implanted GaAs. They used $3 \mathrm{MeV}$ energy to implant $\mathrm{N}^{+}$ions into GaAs substrates with an ion concentration dose of $3 \times 10^{12} \mathrm{~cm}^{-2}$. The $\mathrm{N}^{+}$-implanted GaAs photomixers exhibit improvements in the output power in comparison with their counterparts, photomixers fabricated on low-temperature-grown GaAs. The maximal output power was $2.64 \mu \mathrm{W}$ at $850 \mathrm{GHz}$. No saturation of the output power with increased bias voltage and optical input power was observed. These characteristics make $\mathrm{N}^{+}$-implanted GaAs the material of choice for efficient high power sources of terahertz radiation. (c) 2006 American Institute of Physics.
\end{abstract}

[DOI: $10.1063 / 1.2337523$ ]

The low-temperature-grown GaAs (LT-GaAs) fabricated by molecular-beam epitaxy has been recognized for more than a decade for its subpicosecond photocarrier trapping time and acceptable carrier mobility, and so it is the mostly used material for tunable terahertz sources. ${ }^{1,2}$ However, efficiency limits of the LT-GaAs material are already reached and therefore further performance improvement of photomixer devices can be obtained only by alternative material systems with subpicosecond carrier lifetime. One approach is the implantation of various ions, such as $\mathrm{O}, \mathrm{Si}, \mathrm{Ga}$, and $\mathrm{As}$, into GaAs. ${ }^{3,4}$ This approach has been used to reduce the photocarrier trapping time and thus to achieve high-speed and broad-band performances of GaAs-based photodetector and photomixer devices. ${ }^{5,6}$ Recently the implantation of nitrogen into GaAs is extensively studied, where the main attention is paid to low-energy and high-dose implantation. Beside investigations and applications of GaAs: $\mathrm{N}^{7,8}$ the preparation of $\mathrm{GaN}_{x} \mathrm{As}_{1-x}$ (Refs. 9 and 10) compounds is claimed. In our previous work, we have presented ultrafast photodetectors based on $\mathrm{N}^{+}$-implanted GaAs with increased responsivity and very low dark currents ${ }^{11}$ as well as high-performance GaAs:N bow tie antenna photomixers ${ }^{12}$ as an alternative to the commonly used LT-GaAs based devices. Implantation

\footnotetext{
${ }^{\text {a) }}$ Author to whom correspondence should be addressed; electronic mail: m.mikulics@fz-juelich.de

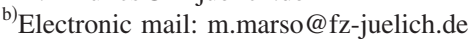

energies of 500,700 , and $880 \mathrm{keV}$ and ion dose of 3 $\times 10^{12} \mathrm{~cm}^{-2}$ were used. Beside material considerations for photomixer improvement, power and frequency performance of the commonly used small-area interdigitated metalsemiconductor-metal (MSM) photodetectors are limited by the $R C$ time constant and the heating of the devices. One possibility to overcome these limitations is the use of a largearea interdigitated traveling-wave photomixer design. ${ }^{13}$ The purpose of this letter is to describe the fabrication process, as well as the properties of such large-area traveling-wave photomixers fabricated on high energy $3 \mathrm{MeV} \mathrm{N} \mathrm{N}^{+}$-implanted GaAs substrates.

The fabrication process started with implantation of $3 \mathrm{MeV}$ energy nitrogen ions into semi-insulating GaAs (001) substrates. For all samples, the ion dose was $3 \times 10^{12} \mathrm{~cm}^{-2}$ without annealing. Next, traveling-wave photomixer structures were fabricated using electron-beam lithography and/or standard photolithography, metallization, and lift-off process, as it was reported in our previous work. ${ }^{13}$ The photomixers consisted of a coplanar stripline with interdigitated MSM finger contacts, integrated with a $70 \Omega$ bow tie antenna and contact pads. A standard Ti/ Au 50/600 nm layer system was used for metallization. The coplanar stripline was $4 \mu \mathrm{m}$ wide. The finger contacts were integrated with the stripline over a length of $200 \mu \mathrm{m}$, with finger width and spacing of 0.5 and $1.4 \mu \mathrm{m}$, respectively. Thus, the described travelingwave photomixers exhibited a large mixing area with more 


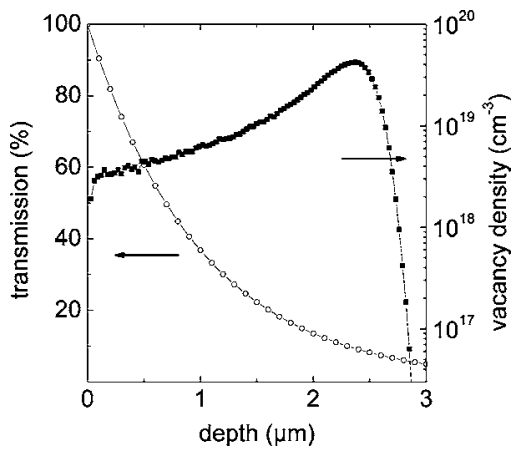

FIG. 1. Depth dependence of induced vacancies calculated using TRIM software for the $\mathrm{N}$ ions implanted into GaAs by implantation energy and dose of $3 \mathrm{MeV}$ and $3 \times 10^{12} \mathrm{~cm}^{-2}$, respectively, and calculated transmission of $780 \mathrm{~nm}$ wavelength vs depth in GaAs.

than hundred finger contacts compared to conventional small-area devices. For the sake of comparison, photomixers with the same geometry as described above were fabricated also on LT-GaAs material grown by conventional molecular beam epitaxy (MBE) process at $275^{\circ} \mathrm{C}$ and annealed in situ at $600{ }^{\circ} \mathrm{C} .{ }^{14}$

Figure 1 shows the vacancy density in the volume of the GaAs for $3 \mathrm{MeV}$ nitrogen ion with a dose of $3 \times 10^{12} \mathrm{~cm}^{-2}$, calculated using the transport range of ions in matter TRIM software. ${ }^{15}$ The vacancy distribution profile is flat over the depth of $\sim 2.5 \mu \mathrm{m}$ and the vacancy density is relatively high, with peak value about $4 \times 10^{19} \mathrm{~cm}^{-3}$. The reason for the use of such high implantation energy is that we need to modify also deeper GaAs regions, because of the large penetration depth of $780 \mathrm{~nm}$ wavelength light, as depicted in Fig. 1 by the transmission versus depth relation. It should be noted that such high implantation energy is a border case: a larger implantation depth would give no further improvement because the applied electric field strongly diminishes with depth. This sets a limit for the efficient collection of photogenerated carriers in deeper regions of the photomixer.

The terahertz power spectrum was measured with a calibrated magnetically tuned $4.2 \mathrm{~K}$ InSb-bolometer, which has a cutoff around $1.4 \mathrm{THz}$. Two heterodyned laser beams with different frequencies $(\sim 780 \mathrm{~nm}$ wavelength) were used for photomixer pumping. ${ }^{13}$ Figure 2 compares the output power versus frequency characteristics of a photomixer based on

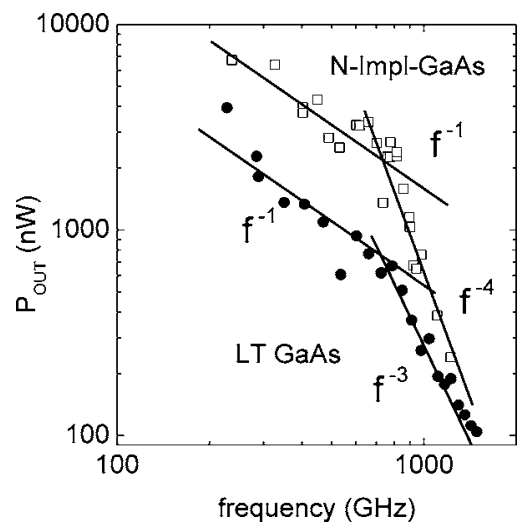

FIG. 2. Terahertz output power spectrum for a traveling-wave photomixer with surface interdigitated MSM contacts of spacing $s=1.4 \mu \mathrm{m}$ fabricated on $3 \mathrm{MeV}$ nitrogen implanted GaAs and LT GaAs (focus length of $150 \mu \mathrm{m}$ full width at half maximum at input wavelength of $780 \mathrm{~nm}$, input power of $400 \mathrm{~mW}$, and bias voltage of $15 \mathrm{~V}$ ).

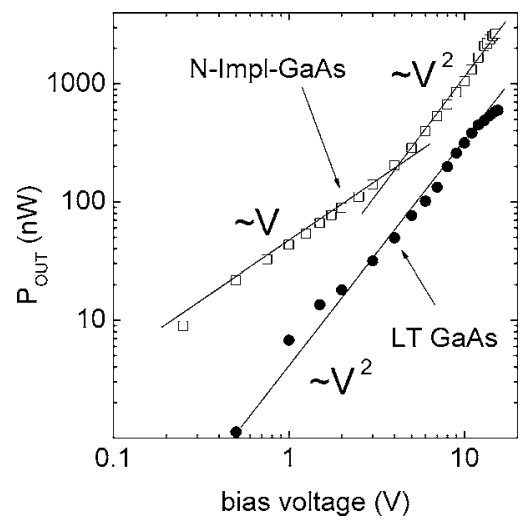

FIG. 3. Output power at $850 \mathrm{GHz}$ vs bias voltage for a traveling-wave photomixer on $3 \mathrm{MeV}$ nitrogen implanted GaAs and LT GaAs. Optical input power $=400 \mathrm{~mW}$.

nitrogen implanted GaAs with one on LT-GaAs material. We used the same LT-GaAs reference photomixer sample as reported previously. ${ }^{14}$ The total input power was $400 \mathrm{~mW}$ and the bias voltage was $15 \mathrm{~V}$. The nitrogen implanted GaAs photomixer exhibits about three times higher output power in the frequency range up to $800 \mathrm{GHz}$, compared with those based on LT-GaAs. We attribute this result to the properties of the $\mathrm{N}^{+}$-implanted material because of its unique mechanism of defect generation during implantation. While arsenic precipitates in LT-GaAs act as deep donors, ${ }^{16}$ the nitrogen implantation process produces significant lattice-expansion defects, ${ }^{7}$ resulting in desired crystallographic, physical, energetic, and electrical properties of the implanted material. The output power decreases for both materials with an increase of the photomixing frequency up to $\sim 800 \mathrm{GHz}$ with factor $f^{-1}$. For higher frequency range the output power of the nitrogen implanted sample decreases more rapidly with $f^{-4}$, compared to $f^{-3}$ for LT-GaAs material. However, the output power of the implanted devices is still more than two times higher in the whole frequency range than for the counterpart on LT-GaAs and about five times higher than values reported previously on traveling-wave devices. ${ }^{17}$ The difference between the devices based on implanted and on lowtemperature grown material can be explained by a longer carrier lifetime of the implanted material. Photoconductive gain and therefore the output power of photomixers are proportional to the lifetime of the photoexcited carriers, ${ }^{1}$ resulting in the improved performance of the implanted device for low frequencies, at the cost of a faster decrease at high frequencies.

Figure 3 shows the output power as a function of the bias voltage for a photomixing frequency of $850 \mathrm{GHz}$. Better performance of the nitrogen implanted GaAs photomixer in the whole range of applied bias voltage is evident. For lower biases (up to $\sim 4 \mathrm{~V}$ ) a more than $300 \%$ increase of the output power is obtained, when compared to the LT-GaAs photomixer, with linear dependence on the bias voltage. At higher biases the output power increases quadratically with the bias voltage for both materials, according to the theory. ${ }^{1}$ The output power of the nitrogen implanted GaAs based photomixer at the highest applied bias level $(15 \mathrm{~V})$ and $850 \mathrm{GHz}$ is $2.64 \mu \mathrm{W}$, which is more than three times higher than that for LT GaAs. Further, no indications of the output power saturation that would be typical for LT-GaAs photomixers are observed. This difference can be attributed to the different 


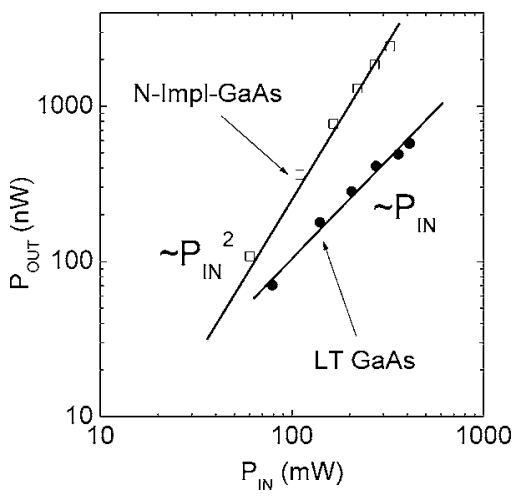

FIG. 4. Output power at $850 \mathrm{GHz}$ vs input power for a traveling-wave photomixer on $3 \mathrm{MeV}$ nitrogen implanted $\mathrm{GaAs}$ and LT GaAs. Bias voltage $=15 \mathrm{~V}$.

origin of the trapping mechanism. Such a different behavior is also seen in Fig. 4, which depicts the output power as function of the optical input power. In the whole measurement range, the photomixer on nitrogen implanted GaAs shows a square dependence of the output power on the optical input power, compared to the linear dependence of the conventional LT-GaAs device. We, again, attribute this superior behavior of the implanted material to the different physical origin of the implantation defects that act as carrier traps, as compared to LT GaAs.

To conclude, we have fabricated terahertz bandwidth photomixers based on $3 \mathrm{MeV}$ nitrogen implanted GaAs. The different origin of the carrier traps, formed during the implantation process, as compared to As precipitates in LT GaAs, leads to the ultrashort carrier lifetime. As compared to the photomixers fabricated on LT GaAs, the devices show 200\% higher output power in the whole frequency range and up to $300 \%$ enhancement at $850 \mathrm{GHz}$ for higher bias levels. The output power of $2.64 \mu \mathrm{W}$ was obtained at $850 \mathrm{GHz}$ and an input power of $400 \mathrm{~mW}$ and $15 \mathrm{~V}$ bias voltage. The GaAs:N interdigitated traveling-wave photomixers show no evidence of output power saturation that are typical for the LT- GaAs-based photomixers. All these ad- vantages are accompanied by the potentially higher attraction in industrial applications mainly for low cost reasons and high reproducibility of material parameters for implantation technology in comparison with the low-temperature MBE process. A further improvement of the device performance is expected by the next optimization of the dose and energy of the nitrogen ions implanted into the GaAs material.

${ }^{1}$ E. R. Brown, F. W. Smith, and K. A. McIntosh, J. Appl. Phys. 73, 1480 (1993).

${ }^{2}$ E. Peytavit, S. Arscott, D. Lippens, G. Mouret, S. Matton, P. Masselin, R. Bocquet, J. F. Lampin, L. Desplanque, and F. Mollot, Appl. Phys. Lett. 81, 1174 (2002)

${ }^{3}$ B. Salem, D. Morris, V. Aimez, J. Beerens, J. Beauvais, and D. Houde, J. Phys.: Condens. Matter 17, 7327 (2005).

${ }^{4}$ A. Krotkus and J.-L. Coutaz, Semicond. Sci. Technol. 20, S142 (2005).

${ }^{5}$ Tze-An Liu, M. Tani, M. Nakajima, M. Hangyo, and Ci-Ling Pan, Appl. Phys. Lett. 83, 1322 (2003).

${ }^{6}$ B. Salem, D. Morris, V. Aimez, J. Beauvais, and D. Houde, Semicond. Sci. Technol. 21, S283 (2006).

${ }^{7}$ J. F. Chen, J. S. Wang, M. M. Huang, and N. C. Chen, Appl. Phys. Lett. 76, 2283 (2000)

${ }^{8}$ J. Wang, H. Mao, Z. Zhu, Q. Zhao, Z. Zhifeng, and W. Lu, Appl. Surf. Sci. 225, 2186 (2005).

${ }^{9}$ K. M. Yu, W. Walukiewicz, J. W. Beeman, M. A. Scarpulla, O. D. Dubon, M. R. Pillai, and M. J. Aziz, Appl. Phys. Lett. 80, 3958 (2002).

${ }^{10}$ S. Sinning, T. Dekorsy, and M. Helm, IEE Proc.: Optoelectron. 151, 361 (2004).

${ }^{11}$ M. Mikulics, M. Marso, P. Kordoš, S. Stanček, P. Kováč, X. Zheng, S. Wu, and R. Sobolewski, Appl. Phys. Lett. 83, 1719 (2003).

${ }^{12}$ M. Mikulics, M. Marso, I. Cámara Mayorga, R. Güsten, S. Stanček, P. Kováč, S. Wu, X. Li, M. Khafizov, R. Sobolewski, E. A. Michael, R. Schieder, M. Wolter, D. Buca, A. Förster, P. Kordoš, and H. Lüth, Appl. Phys. Lett. 87, 041106 (2005).

${ }^{13}$ E. A. Michael, B. Vowinkel, R. Schieder, M. Mikulics, M. Marso, and P. Kordoš, Appl. Phys. Lett. 86, 111119 (2005).

${ }^{14}$ M. Mikulics, E. A. Michael, R. Schieder, J. Stutzki, R. Güsten, M. Marso, A. van der Hart, H. P. Bochem, H. Lüth, and P. Kordoš, Appl. Phys. Lett. 88, 041118 (2006).

${ }^{15}$ J. F. Ziegler, J. P. Biersack, and U. Littmark, The Stopping and Range of Ions in Solids (Pergamon, New York, 1985), Vol. 1, p. 233; see also http:// www.srim.org/

${ }^{16}$ N. Zamdmer and Q. Hu, Appl. Phys. Lett. 75, 2313 (1999).

${ }^{17}$ S. Matsuura, G. A. Blake, R. A. Wyss, J. C. Pearson, C. Kadow, A. W. Jackson, and A. C. Gossard, Appl. Phys. Lett. 74, 2872 (1999). 